\title{
Improved Information Outage Rate in Certain MIMO Systems
}

\author{
David M. Rankin, Member, IEEE, Desmond P. Taylor, Fellow, IEEE, and Philippa A. Martin, Member, IEEE
}

\begin{abstract}
We propose a simple class of encoding/decoding techniques that can be used to improve the information outage rate of certain multiple-input-multiple-output (MIMO) systems. Gains in outage rate can be achieved at low to moderate signal-to-noise ratios in MIMO systems that have fewer receive antennas than transmit antennas. This performance improvement is due to extra "virtual" receive antennas that are created with low-complexity signal processing. A simple space-time block code system using virtual receive antennas is also investigated.
\end{abstract}

Index Terms-Complementary cumulative distribution function (CCDF), information outage rate, multiple-input-multiple-output (MIMO), space-time block codes (STBCs).

\section{INTRODUCTION}

W E propose a simple class of encoding/decoding techniques that can be used to improve the information outage rate of certain multiple-input-multiple-output (MIMO) systems. In particular, the gains are greatest when $n_{t}$ is an integer multiple of $n_{r}$, where $n_{t}$ and $n_{r}$ are the number of transmit and receive antennas, respectively. In order to improve the information outage rate, we require independently faded received signals. This can be achieved by assuming fast fading or time-interleaving of a slow fading process or, alternatively, by using different frequency components in a frequency-selective fading channel. It will be shown that our encoding/decoding technique can significantly increase the information outage rate. Furthermore, this improvement occurs at the outage probabilities of interest, typically $1 \%$ outage rates.

The encoding and decoding technique we employ consists of nothing more than repeatedly transmitting the same data together with stacking the received signal vectors. Fundamentally, we are creating an augmented MIMO channel that has more "virtual" receivers than the original channel. Note that this is equivalent to transmitting a generalized repetition code in the space-time block code (STBC) sense [1]. However, our decoder is quite different from the traditional decoder [1]. Specifically, we combine the received signals in such a way as to increase the effective rank of the MIMO channel. In the limiting case, where we increase the number of virtual receivers to be greater than or equal to the number of transmit antennas, this technique allows us to use VBLAST or some other low-complexity layered space-time decoders that typically require $n_{r} \geq n_{t}$ [2], [3].

Manuscript received December 18, 2005; revised January 16, 2006. This work was supported in part by the New Zealand Foundation for Research, Science, and Technology. The associate editor coordinating the review of this manuscript and approving it for publication was Dr. Xiang-Gen Xia.

The authors are with the Department of Electrical and Computer Engineering, University of Canterbury, Christchurch 8020, New Zealand (e-mail: taylor@elec.canterbury.ac.nz; p.martin@elec.canterbury.ac.nz).

Digital Object Identifier 10.1109/LSP.2006.871863
Similar channel matrix augmentation techniques have been considered in several contexts, such as RAKE-based W-CDMA [4] and in the creation of extended MIMO systems from multipath channels [5]. Ad-hoc networking has also been used to create augmented MIMO channels [6]. However, our aim is to improve the information outage rate of the channel, using simple signal processing techniques.

Our channel augmentation technique entails a loss in achievable rate that is, at best, equivalent to the gain in channel rank. Consequently, it will be shown that the ergodic capacity is reduced by a small amount. However, the improved slope of the information outage rate curves leads to better performance at the outage probabilities of interest.

In Section II, we explain how virtual receivers are created, and in Section III, we show how the information outage rate is calculated. Results are presented in Section IV, and a discussion is included in Section V. A simple STBC scheme using virtual receivers is described in Section VI. Finally, conclusions are drawn in Section VII.

\section{Creating Virtual Receivers}

In the sequel, we use the standard uncorrelated MIMO fast Rayleigh fading channel model with a received vector $\mathbf{y}$

$$
\mathbf{y}=\mathbf{H x}+\mathbf{n}
$$

where $\mathbf{x}$ is the transmitted data, $\mathbf{H}$ is the $n_{r} \times n_{t}$ channel matrix, and $\mathbf{n}$ is the noise vector. Both $\mathbf{H}$ and $\mathbf{n}$ have independently and identically distributed (i.i.d.) elements drawn randomly from $\mathcal{C N}(0,1)$, a complex zero-mean Gaussian distribution with unit variance. Note that both $\mathbf{H}$ and $\mathbf{n}$ are assumed to be independently realized in each time slot. ${ }^{1}$ Furthermore, it is assumed that $\mathbf{H}$ is known to the receiver. We constrain the total transmit power $P$, such that $\mathcal{E}\left[\mathbf{x}^{H} \mathbf{x}\right] \leq P$ and have $\mathcal{E}\left[\mathbf{n n}^{H}\right]=\mathbf{I}$, where $\mathbf{I}$ is the identity matrix. Note that $(\cdot)^{H}$ denotes the Hermitian, or conjugate transpose operator and $\mathcal{E}$ denotes the expectation operator.

In order to increase the effective number of receivers, we simply stack received vectors on top of each other. This technique requires that we transmit the same data in two or more time slots. For example, if we repeat $\mathbf{x}$ in two time slots, we receive

$$
\begin{aligned}
& \mathbf{y}_{1}=\mathbf{H}_{1} \mathbf{x}+\mathbf{n}_{1} \\
& \mathbf{y}_{2}=\mathbf{H}_{2} \mathbf{x}+\mathbf{n}_{2}
\end{aligned}
$$

where $\mathbf{H}_{1}, \mathbf{H}_{2}, \mathbf{n}_{1}$, and $\mathbf{n}_{2}$ are all independent.

\footnotetext{
${ }^{1}$ The model is equally valid for different frequency slots in a frequency-selective fading channel, such as when OFDM is used.
} 
Then stacking the received vectors to form $\mathbf{z}$, produces

$$
\mathbf{z}=\left[\begin{array}{l}
\mathbf{y}_{1} \\
\mathbf{y}_{2}
\end{array}\right]=\left[\begin{array}{l}
\mathbf{H}_{1} \\
\mathbf{H}_{2}
\end{array}\right] \mathbf{x}+\left[\begin{array}{l}
\mathbf{n}_{1} \\
\mathbf{n}_{2}
\end{array}\right]=\mathbf{G x}+\mathbf{v}
$$

where $\mathbf{G}$ now has size $2 n_{r} \times n_{t}$, and the signal-to-noise ratio $(S N R)$ is unchanged (since $\mathcal{E}\left[\mathbf{v}^{H} \mathbf{v}\right]=\mathbf{I}$ and $\mathcal{E}\left[\mathbf{x}^{H} \mathbf{x}\right] \leq P$ ). Thus, if $n_{r} \leq n_{t} / 2$, we have doubled the channel rank. However, because we have transmitted the same data twice, we do not improve the ergodic capacity. In fact, simulations show that the ergodic capacity is slightly reduced. Note that $\mathbf{H}_{1}$ and $\mathbf{H}_{2}$ should be independent in order to maximize both the outage rate and ergodic capacity. The aim is to produce a system with an equal number of transmit and virtual receive antennas, ${ }^{2}$ in order to maximize the improvement in outage information rate.

\section{IMPROVING THE INFORMATION OUTAGE RATE}

It is relatively well known that a larger channel matrix results in a steeper complementary cumulative distribution function $(C C D F)$ in the "roll-off" region [2], [7]. This indicates that we should make the rank of the "effective" channel matrix as large as possible (subject to decoding complexity constraints) in order to maximize the outage rate at the outage probabilities of interest. In our case, with $n_{t}>n_{r}$, this is equivalent to generating more virtual receivers. Note that this improvement in outage rate is more evident at low outage probabilities (say, 1 in 100 blocks in error) than at higher outage probabilities (1 in 10 blocks in error). In fact, at high, and essentially unusable, outage probabilities, the outage capacity can actually decrease with increasing numbers of virtual receive antennas.

The virtual receiver technique increases the size and rank of the augmented channel matrix with a corresponding decrease in data rate. Numerical results in Section IV show that the larger channel matrix results in an improved information outage rate at SNRs and target outage probabilities of interest. The following lemma is used to calculate the information outage rate.

Lemma 1: The information rate of the augmented channel, $\mathbf{G}$, known only at the receiver, is given by

$$
\mathcal{R}=\frac{1}{L} \log _{2}\left(\operatorname{det}\left(\mathbf{I}+\frac{P}{n_{t}} \mathbf{G G}^{H}\right)\right)
$$

where $L$ is the number of blocks combined to create the required virtual receivers.

Proof: We generate a virtual received vector

$$
\mathbf{z}=\bigvee_{i=1}^{L} \mathbf{y}_{i}
$$

where $\bigvee$ is the "stacking" operator. Clearly the transmitter must transmit $L$ copies of $\mathbf{x}$, and therefore, the pre-log factor is given by $1 / L$, the SNR is unchanged and so is the number of transmit antennas. Since the augmented channel matrix contains independent entries, the maximum achievable rate follows directly from [2] and [7]. QED.

\footnotetext{
${ }^{2}$ Simulations suggest that at high SNR we may not want to extend the channel this far due to the reduction in ergodic capacity.
}

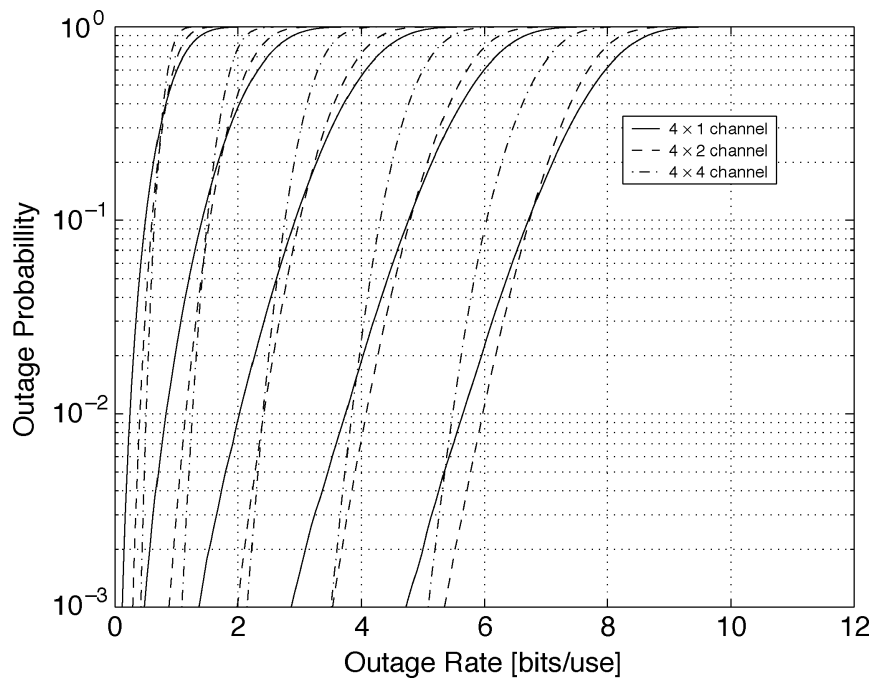

Fig. 1. Outage probability versus information outage rate for a MISO system with and without channel augmentation. SNR $=0,6,12,18,24 \mathrm{~dB}$ from left to right.

Note that we could also increase the number of virtual transmit antennas by simply adding together independently faded blocks of information. However, it can be shown that this does not provide the same improvements as the virtual receive antennas. Furthermore, attempting to increase both the number of virtual transmit and virtual receive antennas will actually make the performance worse. This is because the pre-log factor in the rate calculations decreases faster than the rank of the channel matrix can increase.

\section{RESULTS}

In this section, we consider a simple multiple-input-singleoutput (MISO) channel. Initially, we fix the system with four transmit antennas and one receive antenna. The simulation results are shown in Figs. 1 and 2. The outage probability is plotted against the information outage rate in Fig. 1 for three different channels at a number of different SNRs. The first channel is the $4 \times 1$ MISO channel, while the second and third channels are 4 $\times 2$ and $4 \times 4$ augmented channels created from the original 4 $\times 1$ MISO channel using the virtual receiver technique.

It can be seen from Fig. 1 that the outage rate, at an outage probability of 0.01 , can be improved by a significant amount. Indeed, at low SNR, the outage rate is almost doubled. The relative improvement decreases with increasing SNR, and eventually, at an SNR of $12 \mathrm{~dB}$, the performance of the $4 \times 4$ system equals that of the $4 \times 2$ system. Thereafter, it is more efficient to use the $4 \times 2$ system. This effect is due to the reduction in ergodic capacity, as shown in Fig. 2. Eventually, at a high enough SNR, the augmented channel will not improve the outage rate. However, at most useful values of SNR, the outage rate can be improved. Furthermore, as the required outage probability decreases, our channel augmentation technique significantly increases the relative performance improvement.

The second set of simulation results varies the number of transmit antennas in a MISO system at a fixed SNR of $6 \mathrm{~dB}$, 


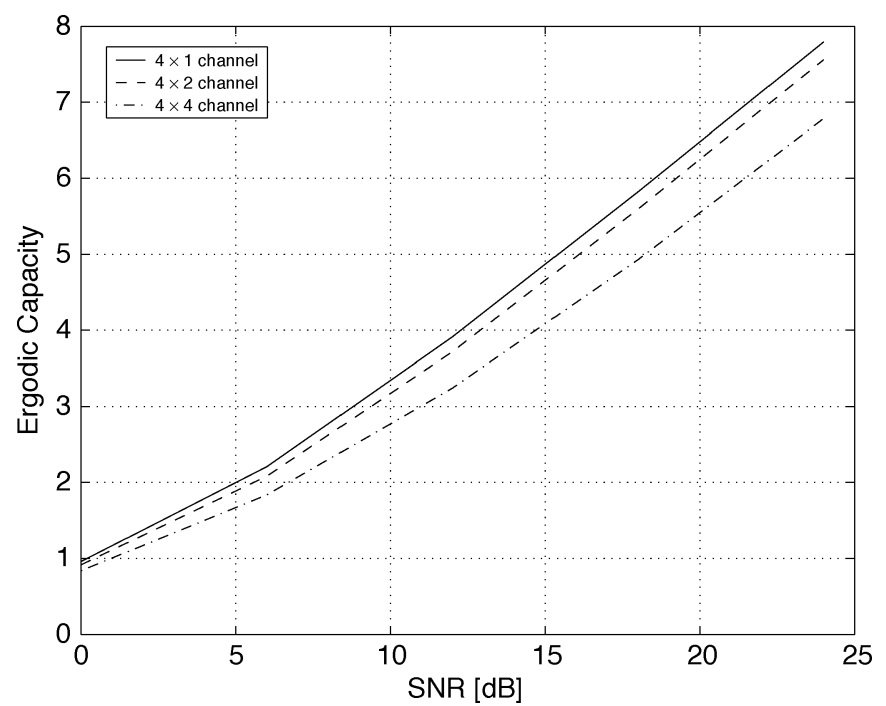

Fig. 2. Ergodic capacity for a MISO system with and without channel augmentation as a function of SNR.

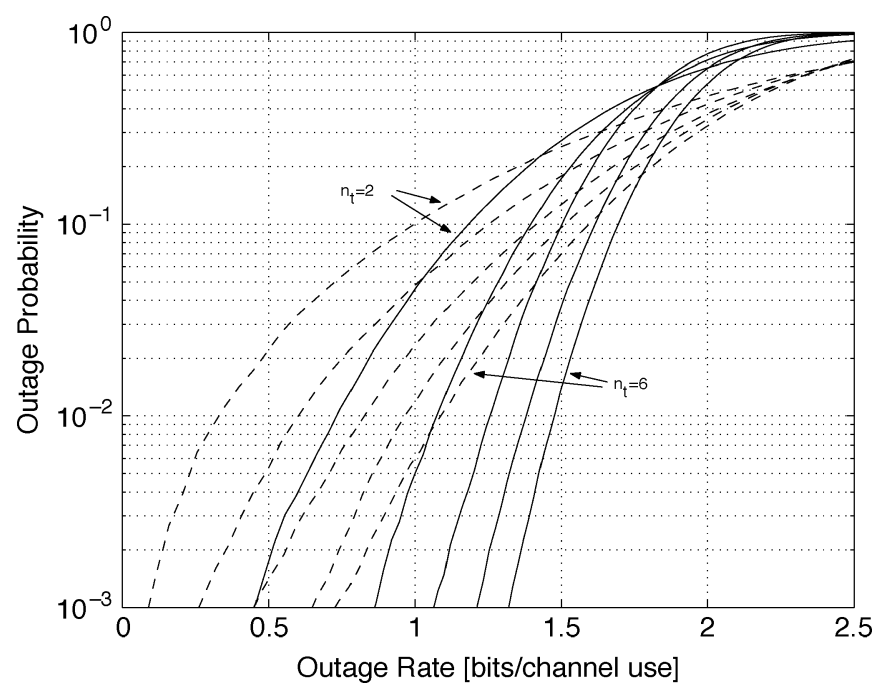

Fig. 3. Outage probability versus information outage rate for a MISO system with an SNR of $6 \mathrm{~dB}$. The number of transmit antennas, $n_{t}=2,3,4,5,6$ is increasing from left to right. The MISO outage capacities are shown as dashed lines. The best augmented channel information outage rates, for an outage probability of 0.01 , are shown as solid lines.

as shown in Fig. 3. It should be noted that the optimal ${ }^{3}$ number of augmented receivers varies with the target outage probability. However, at an outage probability of 0.01 , the optimal number of augmented receivers is $n_{r}^{\text {aug }}=2,3,4,4,4$ for $n_{t}=2,3$, $4,5,6$, respectively, and this corresponds to the results plotted in Fig. 3. The improvement in information outage rate, $\Delta \mathcal{R}$, in this particular scenario is given by $\Delta \mathcal{R}=0.40,0.47,0.43$,

\footnotetext{
${ }^{3}$ This optimality assumes that the total transmitted power is shared equally between all transmit antennas. It has been conjectured, and shown (in the MISO channel) by Telatar [7], that reducing the number of active transmit antennas can improve the information outage rate at very high outage probabilities. In fact, there is an interesting interplay between Telatar's method and adding more augmented receivers. Specifically, Telatar's technique improves the outage rate at high outage probabilities and worsens the outage rate at lower outage probabilities. The augmented receiver technique does the opposite. Thus, the best technique to use will depend on the target outage probability and SNR and may involve a combination of techniques.
}

$0.41,0.39 \mathrm{bits} / \mathrm{channel}$ use. Clearly, this is a notable improvement over the standard system, doubling the information outage rate for small $n_{t}$. Moreover, $\Delta \mathcal{R}$ increases significantly as the target outage probability decreases.

\section{DISCUSSION}

An interesting open problem is the optimal techniques used to create the virtual receivers. Currently, the same data are transmitted multiple times. As mentioned previously, this is a generalized repetition code. Therefore, the question is, can we use a better code? Specifically, if we have multiple possible transmission patterns, we could transmit extra information at the expense of Euclidean distance between the codewords. Note that the extra information would usually only equate to a few bits per $L$ time slots since practical systems could not use particularly long "block lengths." Also note that our channel augmentation technique only works well for uncorrelated, non-line-of-sight Rayleigh fading channels.

One particularly interesting application of the channel augmentation technique would be in an OFDM quasi-static, frequency-selective channel. In this case, we would have access to independently faded MIMO channels with no extra delay penalties. Thus, our technique may be used to improve performance with relatively little extra processing, especially at low SNRs.

A further useful application occurs in some mobile scenarios where maintaining low outage probability is more important than achieving high data rate. In such instances, $n_{r}$, the number of physical receive antennas in the mobile terminal, is often limited to one.

\section{STBC System With VIRTUAL RECEIVERS}

We now show how the proposed virtual receiver can be used to improve performance when only one receive antenna is available. Note this technique can be used in many other scenarios. We consider a simple MIMO system using $n_{t}=2, n_{r}=1$ and the Alamouti STBC [8], where $\mathbf{x}$ is now a STBC codeword spanning $n_{t}=2$ transmit antennas and $T=2$ time slots. We then apply the virtual receiver technique on a block basis (the vectors in (4) become matrices). The STBC can be represented by the matrix

$$
\mathbf{x}=\left[\begin{array}{cc}
s_{1} & -s_{2}^{*} \\
s_{2} & s_{1}^{*}
\end{array}\right]
$$

where $(\cdot)^{*}$ denotes the complex conjugate, and $s_{1}$ and $s_{2}$ are constellation points. Each row is sent using a different transmit antenna and each column is sent in a different time slot. The vector $\mathbf{z}$ and noise vectors $\mathbf{n}_{1}$ and $\mathbf{n}_{2}$ in (4) become matrices of size $n_{r} \times T$. The STBC matrix is transmitted $L$ times.

The symbol-error-rate (SER) performance of the proposed virtual receiver system using $L=2$ transmissions of $\mathbf{x}$ and quadrature phase shift keying (QPSK) symbols is shown in Fig. 4. The performance of the Alamouti STBC using $L=1$ with $n_{r}=1$ or $n_{r}=2$ is also shown. All simulations use the linear processing decoder of [8] for $n_{r} L$ receive antennas. We assume a quasi-static flat Rayleigh fading channel, which is constant over an STBC codeword (two time slots) and varies 


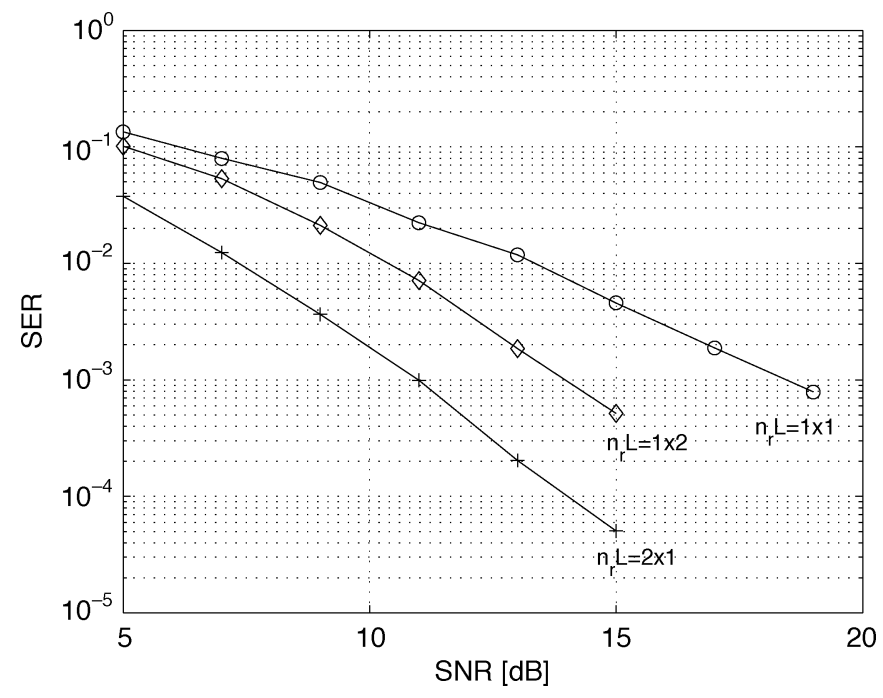

Fig. 4. Performance of the Alamouti STBC using $\left(L=1, n_{r}=1\right)$ and $(L=$ $\left.1, n_{r}=2\right)$ and the virtual receiver using $\left(L=2, n_{r}=1\right)$.

independently between codeword blocks. Each STBC transmission sees a different channel. As expected, we suffer a 3-dB penalty using ( $L=2, n_{r}=1$ ) compared to using ( $L=1$, $\left.n_{r}=2\right)$ due to transmitting the same data twice. However, we obtain the same diversity as $\left(L=1, n_{r}=2\right)$ using $(L=2$, $\left.n_{r}=1\right)$, resulting in a gain of $4.5 \mathrm{~dB}$ at an SER of $10^{-3}$ over $\left(L=1, n_{r}=1\right)$.

\section{CONCLUSION}

The proposed channel augmentation technique can improve the information outage rate of asymmetric MIMO channels under some conditions. At low to moderate SNR and/or low outage probability, this improvement can be significant, doubling the information outage rate in some cases. The cost of this improved performance is typically an increased decoding complexity and a potentially increased decoding delay. In addition, a simple STBC scheme has been simulated using the proposed channel augmentation technique, resulting in improved performance for $n_{t}=2$ and $n_{r}=1$. There remains some interesting open problems regarding the best method to create these virtual receivers.

\section{REFERENCES}

[1] V. Tarokh, N. Seshadri, and A. Calderbank, "Space-time codes for high data rate wireless communication: Performance criterion and code construction," IEEE Trans. Inf. Theory, vol. 44, no. 2, pp. 744-765, Mar. 1998.

[2] G. Foschini and M. Gans, "On limits of wireless communication in a fading environment when using multiple antennas," in Wireless Personal Communications. Norwell, MA: Kluwer, 1998, pp. 311-335, no. 6.

[3] B. Hassibi, "A fast square-root implementation for BLAST," in Proc. Asilomer Conf. Signal Systems Computers, Nov. 2000, pp. 1255-1259.

[4] D. Samardzija, P. Wolniansky, and J. Ling, "Performance evaluation of the VBLAST algorithm in W-CDMA systems," in Proc. VTC_Fall, 2001, pp. 723-727.

[5] A. Ganesan and A. Sayeed, "A virtual input-output framework for transceiver analysis and design for multipath fading channels," IEEE Trans. Commun., vol. 51, no. 7, pp. 1149-1161, Jul. 2003.

[6] M. Dohler, A. Gkelias, and H. Aghvami, "A resource allocation strategy for distributed MIMO multi-hop communication systems," IEEE Commun. Lett., vol. 8, no. 2, pp. 99-101, Feb. 2004.

[7] E. Telatar, "Capacity of multi-antenna Gaussian channels," Eur. Trans. Telecommun., vol. 10, no. 6, pp. 585-596, Nov. 1999.

[8] S. M. Alamouti, "A simple transmit diversity technique for wireless communications," IEEE J. Sel. Areas Commun., vol. 16, no. 8, pp. 1451-1458, Oct. 1998. 